\title{
Existence of Infinitely Many Periodic Solutions for Perturbed Semilinear Fourth-Order Impulsive Differential Inclusions
}

\author{
Massimiliano Ferrara, ${ }^{1}$ Giuseppe Caristi, ${ }^{2}$ and Amjad Salari ${ }^{3}$ \\ ${ }^{1}$ Department of Law and Economics, Mediterranea University of Reggio Calabria, Via dei Bianchi 2, 89131 Reggio Calabria, Italy \\ ${ }^{2}$ Department of Economics, University of Messina, Via dei Verdi 75, 98122 Messina, Italy \\ ${ }^{3}$ Department of Mathematics, Faculty of Sciences, Razi University, Kermanshah 67149, Iran
}

Correspondence should be addressed to Giuseppe Caristi; gcaristi@unime.it

Received 28 October 2015; Accepted 11 February 2016

Academic Editor: Agacik Zafer

Copyright (C) 2016 Massimiliano Ferrara et al. This is an open access article distributed under the Creative Commons Attribution License, which permits unrestricted use, distribution, and reproduction in any medium, provided the original work is properly cited.

This paper discusses the existence of infinitely many periodic solutions for a semilinear fourth-order impulsive differential inclusion with a perturbed nonlinearity and two parameters. The approach is based on a critical point theorem for nonsmooth functionals.

\section{Introduction}

The goal of this paper is to establish the existence of infinitely many periodic solutions for the following perturbed semilinear fourth-order impulsive differential inclusion:

$$
\begin{aligned}
& u^{(\mathrm{iv})}(x)-p u^{\prime \prime}(x)+a(x) u(x) \\
& \quad \in \lambda \partial F(u(x))+\mu \partial G_{u}(x, u(x)), \quad x \in[0, T] \backslash Q, \\
& \Delta\left(u^{\prime \prime}\left(x_{k}\right)\right)=I_{1 k}\left(u^{\prime}\left(x_{k}\right)\right), \\
& -\Delta\left(u^{\prime \prime \prime}\left(x_{k}\right)\right)=I_{2 k}\left(u\left(x_{k}\right)\right), \\
& u(0)=u^{\prime \prime}(0)=u(T)=u^{\prime \prime}(T)=0,2, \ldots, m,
\end{aligned}
$$

where $p$ is a positive constant, $a$ is continuous positive even $2 T$-periodic function on $\mathbb{R}, T>0,0=x_{0}<x_{1}<\cdots<$ $x_{m}<x_{m+1}=T, Q=\left\{x_{1}, x_{2}, \ldots, x_{m}\right\}, \partial F(u)$ and $\partial G_{u}(x, u)$ are generalized gradients of $F$ and $G_{u}$, respectively, the operator $\Delta$ is defined as $\Delta\left(u\left(x_{k}\right)\right):=u\left(x_{k}^{+}\right)-u\left(x_{k}^{-}\right)$, with $u\left(x_{k}^{+}\right)$and $u\left(x_{k}^{-}\right)$denoting the right and left limits, respectively, of $u(x)$ at $x=x_{k}, I_{1 k}, I_{2 k} \in C(\mathbb{R}, \mathbb{R}), k=1,2, \ldots, m, \lambda>0, \mu \geq 0$, and $F: \mathbb{R} \rightarrow \mathbb{R}$ is a locally Lipschitz function satisfying the following:

$(F 1) \quad F(0)=0$ and $\partial F(s)=-\partial F(-s)$ for all $s \in \mathbb{R}$;
(F2) there exist two constants $c>0$ and $r \in[1,2)$, such that

$$
|\xi| \leq c\left(1+|s|^{r-1}\right), \quad \xi \in \partial F(s), s \in \mathbb{R} .
$$

Also, $G$ is a function defined on $[0, T] \times \mathbb{R}$, satisfying the following:

(G1) $G(\cdot, s): \mathbb{R} \rightarrow \mathbb{R}$ is measurable for each $s \in \mathbb{R}, G(x, \cdot)$ : $\mathbb{R} \rightarrow \mathbb{R}$ is locally Lipschitz for $x \in[0, T] \backslash Q, G(x, 0)=$ 0 , and $-\partial G_{s}(-x,-s)=\partial G_{s}(x, s)=\partial G_{s}(x+2 T, s)$ for a.e. $x \in[0, T] \backslash Q$ and $s \in \mathbb{R}$;

(G2) there exists a constant $b>0$, such that

$$
|\xi| \leq b\left(1+|s|^{r-1}\right) \quad s \in \mathbb{R}, \xi \in \partial G_{s}(x, s),
$$

where $r$ is defined in (F2).

We study the existence of solutions, that is, absolutely continuous on every $\left(x_{k}, x_{k+1}\right)$ and left continuous at $x_{k}$ functions which satisfy (1) for a.e. $x$ with (possible) jumps (impulses) at $x_{k}$.

Fourth-order ordinary differential equations act as models for the bending or deforming of elastic beams, and, therefore, they have important applications in engineering and physical sciences. Boundary value problems for fourthorder ordinary differential equations have been of great 
concern in recent years (e.g., see [1-4]). On the other hand, impulsive differential equations occur in many applications such as various mathematical models including population dynamics, ecology, biological systems, biotechnology, industrial robotic, pharmacokinetics, and optimal control. For the general aspects of impulsive differential equations, we refer the reader to [5-9]. In association with this development, a theory of impulsive differential equations has been given extensive attention. Very recently, some researchers have studied the existence and multiplicity of solutions for impulsive fourth-order two-point boundary value problems; we refer the reader to [10-12] and references therein. Differential inclusions arise in models for control systems, mechanical systems, economical systems, game theory, and biological systems to name a few.

Recently, multiplicity of solutions for differential inclusions via nonsmooth variational methods and critical point theory has been considered and here we cite the papers [1319]. For example, in [13] the existence of infinitely many antiperiodic solutions for second-order impulsive differential inclusions has been discussed. In [16], Kristály employing a nonsmooth Ricceri-type variational principle [20], developed by Marano and Motreanu [21], has established the existence of infinitely many radially symmetric solutions for a differential inclusion problem in $\mathbb{R}^{N}$. Also, in [17], the authors extended a recent result of Ricceri concerning the existence of three critical points of certain nonsmooth functionals. Two applications have been given, both in the theory of differential inclusions; the first one concerns a nonhomogeneous Neumann boundary value problem and the second one treats a quasilinear elliptic inclusion problem in the whole $\mathbb{R}^{N}$. Tian and Henderson in [18], based on a nonsmooth version of critical point theory of Ricceri due to Iannizzotto [14], have established the existence of at least three solutions for the a second-order impulsive differential inclusion with a perturbed nonlinearity and two parameters. In [19], three periodic solutions with prescribed wavelength for a class of semilinear fourth-order differential inclusions are obtained by using a nonsmooth version critical point theorem.

In the present paper, motivated by $[13,18,19]$, employing an abstract critical point result (see Theorem 7 below), we are interested in ensuring the existence of infinitely many periodic solutions for problem (1); see Theorem 12. We refer to [22], in which related variational methods are used for nonhomogeneous problems.

To the best of our knowledge, no investigation has been devoted to establishing the existence of infinitely many solutions to a problem such as (1). As one reference on impulsive differential inclusions, we can refer to [23].

A special case of our main result is the following theorem.

Theorem 1. Assume that $(F 1)$ and $(F 2)$ hold, and $I_{i}(0)=0$ and $I_{i}(s) s>0, s \in \mathbb{R}, i=1,2, \ldots, m$. Furthermore, suppose that

$$
\begin{aligned}
& \liminf _{\xi \rightarrow+\infty} \frac{\sup _{|t| \leq \xi} F(t)}{\xi^{2}}=0 \\
& \limsup _{\xi \rightarrow+\infty} \frac{\int_{0}^{1} F\left(\xi\left(x^{2}-x\right)\right) \mathrm{d} x}{(131 / 60) \xi^{2}+\sum_{k=1}^{m} \int_{0}^{\xi\left(2 x_{k}-1\right)} I_{1 k}(s) \mathrm{d} s+\sum_{k=1}^{m} \int_{0}^{\xi\left(x_{k}^{2}-x_{k}\right)} I_{2 k}(s) \mathrm{d} s}=+\infty .
\end{aligned}
$$

Then, the problem

$$
\begin{aligned}
u^{(i v)}(x)-u^{\prime \prime}(x)+u(x) & \in \partial F(u(x)), \quad x \in[0,1] \backslash Q, \\
\Delta\left(u^{\prime \prime}\left(x_{k}\right)\right) & =I_{1 k}\left(u^{\prime}\left(x_{k}\right)\right), \\
-\Delta\left(u^{\prime \prime \prime}\left(x_{k}\right)\right) & =I_{2 k}\left(u\left(x_{k}\right)\right), \\
k & =1,2, \ldots, m, \\
u(0) & =u^{\prime \prime}(0)=u(1)=u^{\prime \prime}(1)=0
\end{aligned}
$$

admits a sequence of classical solutions.

\section{Basic Definitions and Preliminary Results}

Let $\left(X,\|\cdot\|_{X}\right)$ be a real Banach space. We denote by $X^{*}$ the dual space of $X$, while $\langle\cdot, \cdot\rangle$ stands for the duality pairing between $X^{*}$ and $X$. A function $\varphi: X \rightarrow \mathbb{R}$ is called locally Lipschitz if, for all $u \in X$, there exist a neighborhood $U$ of $u$ and a real number $L>0$ such that

$$
|\varphi(v)-\varphi(w)| \leq L\|v-w\|_{X} \quad \forall v, w \in U
$$

If $\varphi$ is locally Lipschitz and $u \in X$, the generalized directional derivative of $\varphi$ at $u$ along the direction $v \in X$ is

$$
\varphi^{\circ}(u ; v):=\limsup _{w \rightarrow u, \tau \rightarrow 0^{+}} \frac{\varphi(w+\tau v)-\varphi(w)}{\tau} .
$$

The generalized gradient of $\varphi$ at $u$ is the set

$$
\partial \varphi(u):=\left\{u^{*} \in X^{*}:\left\langle u^{*}, v\right\rangle \leq \varphi^{\circ}(u ; v) \forall v \in X\right\} .
$$

So $\partial \varphi: X \rightarrow 2^{X^{*}}$ is a multifunction. We say that $\varphi$ has compact gradient if $\partial \varphi$ maps bounded subsets of $X$ into relatively compact subsets of $X^{*}$. 
Lemma 2 (see [24, Proposition 1.1]). Let $\varphi \in C^{1}(X)$ be a functional. Then $\varphi$ is locally Lipschitz and

$$
\begin{gathered}
\varphi^{\circ}(u ; v)=\left\langle\varphi^{\prime}(u), v\right\rangle \quad \forall u, v \in X ; \\
\partial \varphi(u)=\left\{\varphi^{\prime}(u)\right\} \quad \forall u \in X .
\end{gathered}
$$

Lemma 3 (see [24, Proposition 1.3]). Let $\varphi: X \rightarrow \mathbb{R}$ be a locally Lipschitz functional. Then $\varphi^{\circ}(u ; \cdot)$ is subadditive and positively homogeneous for all $u \in X$, and

$$
\varphi^{\circ}(u ; v) \leq L\|v\| \quad \forall u, v \in X,
$$

with $L>0$ being a Lipschitz constant for $\varphi$ around $u$.

Lemma 4 (see [25]). Let $\varphi: X \rightarrow \mathbb{R}$ be a locally Lipschitz functional. Then $\varphi^{\circ}: X \times X \rightarrow \mathbb{R}$ is upper semicontinuous and, for all $\lambda \geq 0, u, v \in X$,

$$
(\lambda \varphi)^{\circ}(u ; v)=\lambda \varphi^{\circ}(u ; v) .
$$

Moreover, if $\varphi, \psi: X \rightarrow \mathbb{R}$ are locally Lipschitz functionals, then

$$
(\varphi+\psi)^{\circ}(u ; v) \leq \varphi^{\circ}(u ; v)+\psi^{\circ}(u ; v) \quad \forall u, v \in X
$$

Lemma 5 (see [24, Proposition 1.6]). Let $\varphi, \psi: X \rightarrow \mathbb{R}$ be locally Lipschitz functionals. Then

$$
\begin{gathered}
\partial(\lambda \varphi)(u)=\lambda \partial \varphi(u) \quad \forall u \in X, \lambda \in \mathbb{R}, \\
\partial(\varphi+\psi)(u) \subseteq \partial \varphi(u)+\partial \psi(u) \quad \forall u \in X .
\end{gathered}
$$

Lemma 6 (see [14, Proposition 1.6]). Let $\varphi: X \rightarrow \mathbb{R}$ be a locally Lipschitz functional with a compact gradient. Then $\varphi$ is sequentially weakly continuous.

We say that $u \in X$ is a (generalized) critical point of a locally Lipschitz functional $\varphi$ if $0 \in \partial \varphi(u)$; that is,

$$
\varphi^{\circ}(u ; v) \geq 0 \quad \forall v \in X
$$

When a nonsmooth functional, $g: X \rightarrow(-\infty,+\infty)$, is expressed as a sum of a locally Lipschitz function, $\varphi: X \rightarrow \mathbb{R}$, and a convex, proper, and lower semicontinuous function, $j: X \rightarrow(-\infty,+\infty)$, that is, $g:=\varphi+j$, a (generalized) critical point of $g$ is every $u \in X$ such that

$$
\varphi^{\circ}(u ; v-u)+j(v)-j(u) \geq 0
$$

for all $v \in X$ (see [24, Chapter 3]).

Henceforth, we assume that $X$ is a reflexive real Banach space, $\mathcal{N}: X \rightarrow \mathbb{R}$ is a sequentially weakly lower semicontinuous functional, $\Upsilon: X \rightarrow \mathbb{R}$ is a sequentially weakly upper semicontinuous functional, $\lambda$ is a positive parameter, $j: X \rightarrow(-\infty,+\infty)$ is a convex, proper, and lower semicontinuous functional, and $D(j)$ is the effective domain of $j$. Write

$$
\begin{aligned}
& \mathscr{M}:=\Upsilon-j, \\
& I_{\lambda}:=\mathscr{N}-\lambda \mathscr{M}=(\mathscr{N}-\lambda \Upsilon)+\lambda j .
\end{aligned}
$$

We also assume that $\mathcal{N}$ is coercive and

$$
D(j) \cap \mathcal{N}^{-1}((-\infty, r)) \neq \emptyset
$$

for all $r>\inf _{X} \mathscr{N}$. Moreover, owing to (17) and provided that $r>\inf _{X} \mathcal{N}$, we can define

$$
\begin{aligned}
\varphi(r) & \\
& :=\inf _{u \in \mathcal{N}^{-1}((-\infty, r))} \frac{\left(\sup _{v \in \mathcal{N}^{-1}((-\infty, r))} \mathscr{M}(v)\right)-\mathscr{M}(u)}{r-\mathscr{N}(u)}, \\
\gamma & :=\liminf _{r \rightarrow+\infty} \varphi(r), \\
\delta & :=\liminf _{r \rightarrow\left(\inf _{X} \mathscr{N}\right)^{+}} \varphi(r) .
\end{aligned}
$$

If $\mathcal{N}$ and $\Upsilon$ are locally Lipschitz functionals, in [22, Theorem 2.1] the following result is proved; it is a more precise version of [21, Theorem 1.1] (see also [20]).

Theorem 7. Under the above assumption on $X, \mathcal{N}$, and $\mathscr{M}$, one has the following:

(a) For every $r>\inf _{X} \mathcal{N}$ and every $\lambda \in(0,1 / \varphi(r))$, the restriction of the functional $I_{\lambda}=\mathcal{N}-\lambda \mathscr{M}$ to $\mathcal{N}^{-1}((-\infty, r))$ admits a global minimum, which is a critical point (local minimum) of $I_{\lambda}$ in $X$.

(b) If $\gamma<+\infty$, then, for each $\lambda \in(0,1 / \gamma)$, the following alternative holds: either

(b1) $I_{\lambda}$ possesses a global minimum or

(b2) there is a sequence $\left\{u_{n}\right\}$ of critical points (local minima) of $I_{\lambda}$ such that $\lim _{n \rightarrow+\infty} \mathscr{N}\left(u_{n}\right)=+\infty$.

(c) If $\delta<+\infty$, then, for each $\lambda \in(0,1 / \delta)$, the following alternative holds: either

(c1) there is a global minimum of $\mathcal{N}$ which is a local minimum of $I_{\lambda}$ or

(c2) there is a sequence $\left\{u_{n}\right\}$ of pairwise distinct critical points (local minima) of $I_{\lambda}$, with $\lim _{n \rightarrow+\infty} \mathcal{N}\left(u_{n}\right)=\inf _{X} \mathcal{N}$, which converges weakly to a global minimum of $\mathcal{N}$.

Now we recall some basic definitions and notations. We consider the reflexive Banach space $X:=H^{2}(0, T) \cap H_{0}^{1}(0, T)$ endowed with the norm

$$
\|u\|=\left(\int_{0}^{T}\left(\left|u^{\prime \prime}(x)\right|^{2}+\left|u^{\prime}(x)\right|^{2}+|u(x)|^{2}\right) \mathrm{d} x\right)^{1 / 2} .
$$

Obviously, $X$ is a reflexive Banach space and completely embedded in $C([0, T])$. So there exists a constant $C_{0}$, such that $C_{0}=\sup _{u \in X,\|u\| \neq 0}\left(\|u\|_{\infty} /\|u\|\right)$. From the positivity of $p$ and $a$, it is easy to see that

$$
\begin{aligned}
& \|u\|_{X}=\left(\int _ { 0 } ^ { T } \left(\left|u^{\prime \prime}(x)\right|^{2}+p\left|u^{\prime}(x)\right|^{2}\right.\right. \\
& \left.\left.\quad+a(x)|u(x)|^{2}\right) \mathrm{~d} x\right)^{1 / 2}
\end{aligned}
$$


is also a norm of $X$, which is equivalent to the usual norm. Therefore, there exist two constants $C_{1}$ and $C_{2}$ such that

$$
C_{1}\|u\| \leq\|u\|_{X} \leq C_{2}\|u\| \text {. }
$$

Thus,

$$
\|u\|_{\infty} \leq \frac{C_{0}}{C_{1}}\|u\|_{X} .
$$

Definition 8. A function $u \in X$ is said to be a weak solution of problem (1) such that, corresponding to it, there exists a mapping $[0, T] \ni x \mapsto u^{*}(x)$ with $u^{*}(x) \in \lambda \partial F(u(x))+$ $\mu \partial G_{u}(x, u(x))$, for a.e. $x \in[0, T]$, and having the property that, for every $v \in X, u^{*} v \in L^{1}[0, T]$ and

$$
\begin{aligned}
& \int_{0}^{T}\left(u^{\prime \prime}(x) v^{\prime \prime}(x)+p u^{\prime}(x) v^{\prime}(x)+a(x) u(x) v(x)\right. \\
& \left.-u^{*}(x) v(x)\right) \mathrm{d} x+\sum_{k=1}^{m} I_{2 k}\left(u\left(x_{k}\right)\right) v\left(x_{k}\right) \\
& +\sum_{k=1}^{m} I_{1 k}\left(u^{\prime}\left(x_{k}\right)\right) v^{\prime}\left(x_{k}\right)=0 .
\end{aligned}
$$

Definition 9. A solution $u$ is called a classical solution of the impulsive differential inclusion (1) if $u \in A C^{3}\left(x_{k}, x_{k+1}\right)$ and $u\left(x_{k}^{-}\right)=u\left(x_{k}\right)$, for $k=1, \ldots, n$, and

$$
\begin{aligned}
& u^{(\mathrm{iv})}(x)+p u^{\prime \prime}(x)+a(x) u(x)=u^{*}(x), \\
& \quad x \in[0, T] \backslash Q, \\
& \Delta u^{\prime \prime}\left(x_{k}\right)=I_{1 k}\left(u^{\prime}\left(x_{k}\right)\right), \quad k=1,2, \ldots, m, \\
& -\Delta\left(u^{\prime \prime \prime}\left(x_{k}\right)\right)=I_{2 k}\left(u\left(x_{k}\right)\right), \\
& u(0)=u^{\prime \prime}(0)=u(T)=u^{\prime \prime}(T)=0,
\end{aligned}
$$

where $u^{*}(x) \in \lambda F(u(x))+\mu G_{u}(x, u(x))$ for a.e. $x \in[0, T]$.

Lemma 10. If $u \in X$ is a weak solution of (1), then $u$ is a classical solution of (1).

Proof. Let $u \in X$ be a weak solution of (1). Then there exists $u^{*}$ with $u^{*}(x) \in \lambda \partial F(u(x))+\mu \partial G_{u}(x, u(x))$ for a.e. $x \in[0, T]$, satisfying (23). Using integration by parts (23) becomes

$$
\begin{aligned}
& \int_{0}^{T} u^{\prime \prime}(x) v^{\prime \prime}(x) \mathrm{d} x \\
& =\int_{0}^{T}\left(p u^{\prime \prime}(x)-a(x) u(x)+u^{*}(x)\right) v(x) \mathrm{d} x \\
& \quad-\sum_{k=1}^{m} I_{2 k}\left(u\left(x_{k}\right)\right) v\left(x_{k}\right) \\
& \quad-\sum_{k=1}^{m} I_{1 k}\left(u^{\prime}\left(x_{k}\right)\right) v^{\prime}\left(x_{k}\right)
\end{aligned}
$$

so $u \in H^{4}((0, T) \backslash Q)$, and (25) holds for each $v \in X$ with $v\left(x_{k}\right)=v^{\prime}\left(x_{k}\right)=0, k=1, \ldots, m$. Through integration by parts, we obtain

$$
\begin{aligned}
u^{(\mathrm{iv})}(x)-p u^{\prime \prime}(x)+a(x) u(x)-u^{*}(x) & =0, \\
\text { a.e. } x & \in[0, T] \backslash Q .
\end{aligned}
$$

Due to $u \in X$, similar to [26, Section 2], we can get $u \in$ $A C^{3}\left(x_{k}, x_{k+1}\right)$ and $u\left(x_{k}^{-}\right)=u\left(x_{k}\right)$ for $k=1, \ldots, n$. Now we show that the boundary conditions are satisfied. Choose any $j \in\{0,1,2, \ldots, m-1\}$ and $v \in X$ such that $v(x)=0$ if $x \in\left[x_{k}, x_{k+1}\right]$ for $k \neq j$. Then, from (25) and by means of integration by parts, we can get

$$
\begin{aligned}
& u^{\prime \prime}\left(x_{j+1}\right) v^{\prime}\left(x_{j+1}\right)-u^{\prime \prime}\left(x_{j}\right) v^{\prime}\left(x_{j}\right) \\
& \quad+\int_{x_{j}}^{x_{j+1}}\left(u^{(\mathrm{iv})}-p u^{\prime \prime}+a(x) u-u^{*}\right) v \mathrm{~d} x=0 .
\end{aligned}
$$

Thus,

$$
\begin{aligned}
& \sum_{j=0}^{m-1}\left(u^{\prime \prime}\left(x_{j+1}\right) v^{\prime}\left(x_{j+1}\right)-u^{\prime \prime}\left(x_{j}\right) v^{\prime}\left(x_{j}\right)\right) \\
& \quad+\sum_{j=0}^{m-1} \int_{x_{j}}^{x_{j+1}}\left(u^{(\mathrm{iv})}-p u^{\prime \prime}+a(x) u-u^{*}\right) v \mathrm{~d} x \\
& =0
\end{aligned}
$$

which implies that

$$
\begin{aligned}
& u^{\prime \prime}(T) v^{\prime}(T)-u^{\prime \prime}(0) v^{\prime}(0) \\
& +\int_{0}^{T}\left(u^{(\text {iv })}(x)-p u^{\prime \prime}(x)+a(x) u(x)-u^{*}(x)\right) \\
& \quad \cdot v(x) \mathrm{d} x=0 .
\end{aligned}
$$

Since $u$ satisfies (26), we have that $u^{\prime \prime}(T) v^{\prime}(T)-u^{\prime \prime}(0) v^{\prime}(0)=0$ holds for all $v \in X$, which implies that $u^{\prime \prime}(0)=u^{\prime \prime}(T)=0$ and in view of $u \in X$ we get $u(0)=u(T)=0$. Now using a technique similar to the technique of [18, Lemma 3.5] shows that the impulsive conditions are satisfied. From the equality

$$
\begin{aligned}
u^{(\mathrm{iv})}(x) v(x)= & \left\{\left\{\int_{0}^{x} u^{(\mathrm{iv})}(s) \mathrm{d} s\right\} v(x)\right)^{\prime} \\
& -\left(\int_{0}^{x} u^{(\mathrm{iv})}(s) \mathrm{d} s\right) v^{\prime}(x),
\end{aligned}
$$


we have

$$
\begin{aligned}
& \int_{0}^{T} u^{(\mathrm{iv})}(x) v(x) \mathrm{d} x=\int_{0}^{T}\left[\left\{\left\{\int_{0}^{x} u^{(\mathrm{iv})}(s) \mathrm{d} s\right\} v(x)\right)^{\prime}\right. \\
& \left.-\left(\int_{0}^{x} u^{(\mathrm{iv})}(s) \mathrm{d} s\right) v^{\prime}(x)\right] \mathrm{d} x=v(T) \\
& \cdot \int_{0}^{T} u^{(\mathrm{iv})}(x) \mathrm{d} x-\int_{0}^{T}\left[u^{\prime \prime \prime}(x)-u^{\prime \prime \prime}(0)\right. \\
& \left.-\sum_{0 \leq x_{k}<x} \Delta u^{\prime \prime \prime}\left(x_{k}\right)\right] v^{\prime}(x) \mathrm{d} x=\left[u^{\prime \prime \prime}(T)\right. \\
& \left.-u^{\prime \prime \prime}(0)-\sum_{k=1}^{m} \Delta u^{\prime \prime \prime}\left(x_{k}\right)\right] v(T)-\int_{0}^{T} u^{\prime \prime \prime}(x) \\
& +v^{\prime}(x) \mathrm{d} x+u^{\prime \prime \prime}(0)[v(T)-v(0)] \\
& +\sum_{k=1}^{m} \Delta u^{\prime \prime \prime}\left(x_{k}\right) v(T)=u^{\prime \prime \prime}(T) v(T)-u^{\prime \prime \prime}(0) v(0) \\
& +\sum_{k=1}^{m} \int_{x_{k}}^{x_{k+1}} \sum_{0 \leq x_{k}<x} \Delta u^{\prime \prime \prime}\left(x_{k}\right) v^{\prime}(x) \mathrm{d} x=u^{\prime \prime \prime}(T) v(T) \\
& +u^{\prime \prime \prime}(0) v(0)-\sum_{k=1}^{m} \Delta u^{\prime \prime \prime}\left(x_{k}\right) v(T)-\int_{0}^{T} u^{\prime \prime}(x) \\
& +v^{\prime \prime}(x) \mathrm{d} x-\sum_{k=1}^{m} \Delta u^{\prime \prime \prime}\left(x_{k}\right) v\left(x_{k}\right)
\end{aligned}
$$

Substituting (31) into (25), we have

$$
\begin{gathered}
\int_{0}^{T}\left(u^{(\mathrm{iv})}(x)-p u^{\prime \prime}(x)+a(x) u(x)-u^{*}(x)\right) \\
\cdot v(x) \mathrm{d} x+u^{\prime \prime \prime}(T) v(T)-u^{\prime \prime \prime}(0) v(0) \\
+\sum_{k=1}^{m}\left[-\Delta u^{\prime \prime \prime}\left(x_{k}\right)-I_{2 k}\left(u\left(x_{k}\right)\right)\right] v\left(x_{k}\right)=0 .
\end{gathered}
$$

Since $u$ satisfies (26), we have

$$
\begin{gathered}
u^{\prime \prime \prime}(T) v(T)-u^{\prime \prime \prime}(0) v(0)-\sum_{k=1}^{m} \Delta\left(u^{\prime \prime \prime}\left(x_{k}\right)\right) v\left(x_{k}\right) \\
-\sum_{k=1}^{m} I_{2 k}\left(u\left(x_{k}\right)\right) v\left(x_{k}\right)=0
\end{gathered}
$$

for all $v \in X$. Thus, $-\Delta u^{\prime \prime \prime}\left(x_{k}\right)=I_{2 k}\left(u\left(x_{k}\right)\right), k=1, \ldots, m$. Similarly from the equality

$$
\begin{aligned}
u^{\prime \prime}(x) v(x)= & \left(\left\{\int_{0}^{x} u^{\prime \prime}(s) \mathrm{d} s\right\} v(x)\right)^{\prime} \\
& -\left(\int_{0}^{x} u^{\prime \prime}(s) \mathrm{d} s\right) v^{\prime}(x),
\end{aligned}
$$

we have $\Delta u^{\prime \prime}\left(x_{k}\right)=I_{1 k}\left(u^{\prime}\left(x_{k}\right)\right)$. So $u$ is a classical solution of (1).

Now we introduce the functionals $\mathcal{N}, \mathscr{F}, \mathscr{G}$, and $I_{\lambda}$ by

$$
\begin{aligned}
\mathcal{N}(u)= & \frac{1}{2}\|u\|_{X}+\sum_{k=1}^{m} \int_{0}^{u^{\prime}\left(t_{\mathrm{k}}\right)} I_{1 k}(s) \mathrm{d} s \\
& +\sum_{k=1}^{m} \int_{0}^{u\left(t_{k}\right)} I_{2 k}(s) \mathrm{d} s, \quad u \in X, \\
\mathscr{F}(u)= & \int_{0}^{T} F(u(x)) \mathrm{d} x, \\
\mathscr{G}(u)= & \int_{0}^{T} G(x, u(x)) \mathrm{d} x,
\end{aligned}
$$

$u \in X$

$$
I_{\lambda, \mu}(u)=\mathscr{N}(u)-\lambda \mathscr{F}(u)-\mu \mathscr{G}(u) \quad u \in X .
$$

Thus, $\mathcal{N} \in C^{1}(X, \mathbb{R})$ and

$$
\begin{aligned}
& \left\langle\mathcal{N}^{\prime}(u), v\right\rangle=\int_{0}^{T}\left(u^{\prime \prime}(x) v^{\prime \prime}(x)+p u^{\prime}(x) v^{\prime}(x)\right. \\
& +a(x) u(x) v(x)) \mathrm{d} x+\sum_{k=1}^{m} I_{2 k}\left(u\left(t_{k}\right)\right) v\left(t_{k}\right) \\
& +\sum_{k=1}^{m} I_{1 k}\left(u^{\prime}\left(t_{k}\right)\right) v^{\prime}\left(t_{k}\right)
\end{aligned}
$$

for all $u, v \in X$.

Lemma 11. Assume that (F1), (F2), (G1), and (G2) hold. Then the functional $I_{\lambda, \mu}: X \rightarrow \mathbb{R}$ is locally Lipschitz. Moreover, each critical point $u \in X$ of $I_{\lambda, \mu}$ is a weak solution of (1).

Proof. Let $I_{\lambda, \mu}=\mathcal{N}(u)+I_{1}(u)$, where $I_{1}(u)=-\lambda \mathscr{F}(u)-$ $\mu \mathscr{G}(u)$. Since $\mathcal{N} \in C^{1}(X, \mathbb{R})$ by Lemma $2, \mathcal{N}$ is locally Lipschitz on $X$. From (F2) and (G2), we know that $I_{1}$ is locally Lipschitz on $L^{r}([0, T])$. Moreover, $X$ is compactly embedded into $L^{r}([0, T])$. Thus, $I_{1}$ is locally Lipschitz on $X[27$, Theorem 2.2]. According to Lemma 5, we get

$$
\begin{aligned}
\partial I_{1}(u) \subset- & \lambda \int_{0}^{T} \partial F(u(x)) \mathrm{d} x \\
& -\mu \int_{0}^{T} \partial G_{u}(x, u(x)) \mathrm{d} x .
\end{aligned}
$$


The explanation of (37) is as follows: for every $u^{*} \in \partial I_{1}(u)$, there is a corresponding mapping $u^{*}(x) \in-\lambda \partial F(u(x))-$ $\mu \partial G_{u}(x, u(x))$ for a.e. $x \in[0, T]$ having the property that, for every $v \in X$, the function $u^{*} v \in L^{1}[0, T]$ and $\left\langle u^{*}, v\right\rangle=$ $\int_{0}^{T} u^{*}(x) v(x) \mathrm{d} x$. Therefore, $I_{\lambda, \mu}$ is locally Lipschitz on $X$.

Now we show that each critical point of $I_{\lambda, \mu}$ is a weak solution of (1). Assume that $u \in X$ is a critical point of $I_{\lambda, \mu}$. So

$$
\begin{aligned}
0 & \in \partial I_{\lambda, \mu}(u) \\
& =\left\{u^{*} \in X^{*}:\left\langle u^{*}, v\right\rangle \leq I_{\lambda, \mu}^{\circ}(u ; v) \text { for } v \in X\right\} .
\end{aligned}
$$

So, by Lemma 2 and (38), we have

$$
\mathcal{N}^{\prime}(u)+u^{*}=0 \quad \text { with } u^{*} \in \partial I_{1}(u)
$$

and hence $u^{*}(x) \in-\lambda \partial F(u(x))-\mu \partial G_{u}(x, u(x))$ a.e. on $[0, T]$. It follows from (37) and (39) that for every $v \in X$ we have

$$
\begin{aligned}
& \int_{0}^{T}\left(u^{\prime \prime}(x) v^{\prime \prime}(x)+p u^{\prime}(x) v^{\prime}(x)+a(x) u(x) v(x)\right. \\
& \left.+u^{*}(x) v(x)\right) \mathrm{d} x+\sum_{k=1}^{m} I_{2 k}\left(u\left(t_{k}\right)\right) v\left(t_{k}\right) \\
& +\sum_{k=1}^{m} I_{1 k}\left(u^{\prime}\left(t_{k}\right)\right) v^{\prime}\left(t_{k}\right)=0
\end{aligned}
$$

for all $v \in X$. Thus, by Definition $8, u$ is a weak solution of (1).

\section{Main Results}

First for every $\xi \in \mathbb{R}^{+}$we set

$$
\begin{aligned}
D(\xi)= & \frac{C_{2}^{2}}{2} \xi^{2}\left(4 T+\frac{T^{3}}{3}+\frac{T^{5}}{30}\right) \\
& +\sum_{k=1}^{m} \int_{0}^{\xi\left(2 x_{k}-T\right)} I_{1 k}(s) \mathrm{d} s \\
& +\sum_{k=1}^{m} \int_{0}^{\xi\left(x_{k}^{2}-T x_{k}\right)} I_{2 k}(s) \mathrm{d} s .
\end{aligned}
$$

Now we formulate our main result using the following assumptions:

(F3) $\liminf \operatorname{in}_{\xi \rightarrow+\infty}\left(\sup _{|t| \leq \xi} F(t) / \xi^{2}\right)<(1 / 2 T)\left(C_{1} / C_{0}\right)^{2}$ $\lim \sup _{\xi \rightarrow+\infty}\left(\int_{0}^{T} F\left(\xi\left(x^{2}-T x\right)\right) \mathrm{d} x / D(\xi)\right) ;$

(I1) $I_{j k}(0)=0, I_{j k}(s) s>0, s \in \mathbb{R}, j=1,2$ and $k=$ $1,2, \ldots, m$.

Theorem 12. Assume that (F1)-(F3) and (I1) hold. Let

$$
\begin{aligned}
& \lambda_{1}:=\frac{1}{\lim \sup _{\xi \rightarrow+\infty}\left(\int_{0}^{T} F\left(\xi\left(x^{2}-T x\right)\right) \mathrm{d} x / D(\xi)\right)}, \\
& \lambda_{2}:=\frac{C_{1}^{2}}{2 T C_{0}^{2} \liminf \inf _{\xi \rightarrow+\infty}\left(\sup _{|t| \leq \xi} F(t) / \xi^{2}\right)} .
\end{aligned}
$$

Then, for every $\lambda \in\left(\lambda_{1}, \lambda_{2}\right)$ and every nonnegative function $G$ satisfying (G1), (G2), and the assumption

$$
\text { (G3) } G_{\infty}:=\lim _{\xi \rightarrow+\infty}\left(\int_{0}^{T} \sup _{|t| \leq \xi} G(x, t) \mathrm{d} x / \xi^{2}\right)<+\infty,
$$

putting

$$
\begin{aligned}
\mu_{G, \lambda}:= & \frac{1}{2 G_{\infty}}\left(\frac{C_{1}}{C_{0}}\right)^{2} \\
& \cdot\left(1-2 T \lambda\left(\frac{C_{0}}{C_{1}}\right)^{2} \liminf _{\xi \rightarrow+\infty} \frac{\sup _{|t| \leq \xi} F(t)}{\xi^{2}}\right),
\end{aligned}
$$

where $\mu_{G, \lambda}=+\infty$ when $G_{\infty}=0$, for every $\mu \in\left[0, \mu_{G, \lambda}\right)$ problem (1) admits an unbounded sequence of classical solutions in $X$.

Proof. Our goal is to apply Theorem $7(\mathrm{~b})$ to (1). For this purpose, we fix $\bar{\lambda} \in\left(\lambda_{1}, \lambda_{2}\right)$ and let $G$ be a nonnegative function satisfying (G1)-(G3). Since $\bar{\lambda}<\lambda_{2}$, we have

$$
\begin{aligned}
& \mu_{G, \bar{\lambda}}=\frac{1}{2 G_{\infty}}\left(\frac{C_{1}}{C_{0}}\right)^{2} \\
& \cdot\left(1-2 T \bar{\lambda}\left(\frac{C_{0}}{C_{1}}\right)^{2} \liminf _{\xi \rightarrow+\infty} \frac{\sup _{|t| \leq \xi} F(t)}{\xi^{2}}\right)>0 .
\end{aligned}
$$

Now fix $\bar{\mu} \in\left(0, \mu_{g, \bar{\lambda}}\right)$, put $\nu_{1}:=\lambda_{1}$, and

$$
\nu_{2}:=\frac{\lambda_{2}}{1+2\left(C_{0} / C_{1}\right)^{2}(\bar{\mu} / \bar{\lambda}) \lambda_{2} G_{\infty}} .
$$

If $G_{\infty}=0$, then $\nu_{1}=\lambda_{1}, \nu_{2}=\lambda_{2}$, and $\bar{\lambda} \in\left(\nu_{1}, v_{2}\right)$. If $G_{\infty} \neq 0$, since $\bar{\mu}<\mu_{G, \bar{\lambda}}$, we have

$$
\frac{\bar{\lambda}}{\lambda_{2}}+2\left(\frac{C_{0}}{C_{1}}\right)^{2} \bar{\mu} G_{\infty}<1,
$$

and so

$$
\frac{\lambda_{2}}{1+2\left(C_{0} / C_{1}\right)^{2}(\bar{\mu} / \bar{\lambda}) \lambda_{2} G_{\infty}}>\bar{\lambda}
$$

to wit, $\bar{\lambda}<v_{2}$. Hence, taking into account that $\bar{\lambda}>\lambda_{1}=v_{1}$, one has $\bar{\lambda} \in\left(\nu_{1}, v_{2}\right)$. Now, set

$$
J(x, s):=F(s)+\frac{\bar{\mu}}{\bar{\lambda}} G(x, s)
$$

for all $(x, s) \in[0, T] \times \mathbb{R}$. Assume that $j$ is exactly zero in $X$ and for each $u \in X$ and put

$$
\begin{aligned}
& \Upsilon(u):=\int_{0}^{T} J(x, u(x)) \mathrm{d} x, \\
& \mathscr{M}(u):=\Upsilon(u)-j(u)=\Upsilon(u), \\
& I_{\bar{\lambda}}(u):=\mathscr{N}(u)-\bar{\lambda} \mathscr{M}(u)=\mathscr{N}(u)-\bar{\lambda} \Upsilon(u) .
\end{aligned}
$$


It is easy to prove that $\mathcal{N}$ is sequentially weakly lower semicontinuous on $X$. Obviously, $\mathcal{N} \in C^{1}(X)$. By Lemma 2, $\mathcal{N}$ is locally Lipschitz on $X$. By Lemma $11, \mathscr{F}$ and $\mathscr{G}$ are locally Lipschitz on $L^{2}([0, T])$. So, $\Upsilon$ is locally Lipschitz on $L^{2}([0, T])$, and since $X$ is compactly embedded into $L^{2}([0, T]), Y$ is locally Lipschitz on $X$. In addition, $\Upsilon$ is sequentially weakly upper semicontinuous. For all $u \in X$, by (I1),

$$
\begin{aligned}
& \int_{0}^{u^{\prime}\left(x_{k}\right)} I_{1 k}(s) \mathrm{d} s>0, \quad k=1,2, \ldots, m, \\
& \int_{0}^{u\left(x_{k}\right)} I_{2 k}(s) \mathrm{d} s>0, \quad k=1,2, \ldots, m .
\end{aligned}
$$

So, we have

$$
\begin{aligned}
\mathcal{N}(u)= & \frac{1}{2}\|u\|_{X}^{2}+\sum_{k=1}^{m} \int_{0}^{u^{\prime}\left(x_{k}\right)} I_{1 k}(s) \mathrm{d} s \\
& +\sum_{k=1}^{m} \int_{0}^{u\left(x_{k}\right)} I_{2 k}(s) \mathrm{d} s>\frac{1}{2}\|u\|_{X}^{2}
\end{aligned}
$$

for all $u \in X$. Hence, $\mathcal{N}$ is coercive and $\inf _{X} \mathcal{N}=\mathcal{N}(0)=0$. Under our hypotheses, we want to show that there exists a sequence $\left\{\bar{u}_{n}\right\} \subset X$ of critical points for the functional $I_{\bar{\lambda}}$; that is, every element $\bar{u}_{n}$ satisfies

$$
I_{\bar{\lambda}}^{\circ}\left(\bar{u}_{n}, v-\bar{u}_{n}\right) \geq 0, \quad \text { for every } v \in X
$$

Now, we prove that $\gamma<+\infty$. For this, let $\left\{\xi_{n}\right\}$ be a sequence of positive numbers such that $\lim _{n \rightarrow+\infty} \xi_{n}=+\infty$ and

$$
\begin{aligned}
& \lim _{n \rightarrow+\infty} \frac{\int_{0}^{T} \sup _{|t| \leq \xi_{n}} J(x, t) \mathrm{d} x}{\xi_{n}^{2}} \\
& \quad=\liminf _{\xi \rightarrow+\infty} \frac{\int_{0}^{T} \sup _{|t| \leq \xi} J(x, t) \mathrm{d} x}{\xi^{2}} .
\end{aligned}
$$

Put

$$
r_{n}:=\frac{1}{2}\left(\frac{C_{1} \xi_{n}}{C_{0}}\right)^{2}, \quad \forall n \in \mathbb{N} .
$$

Then, for all $v \in X$ with $\mathscr{N}(v)<r_{n}$, taking into account that $\|v\|_{X}^{2}<2 r_{n}$ and $\|v\|_{\infty} \leq\left(C_{0} / C_{1}\right)\|v\|_{X}$, one has $|v(x)| \leq \xi_{n}$ for every $x \in[0, T]$. Therefore, for all $n \in \mathbb{N}$,

$$
\begin{aligned}
\varphi\left(r_{n}\right) & =\inf _{u \in \mathcal{N}^{-1}((-\infty, r))} \frac{\left(\sup _{v \in \mathcal{N}^{-1}((-\infty, r))} \mathscr{M}(v)\right)-\mathscr{M}(u)}{r-\mathscr{N}(u)} \\
& \leq \frac{\sup _{\|v\|_{X}^{2}<2 r_{n}}(\mathscr{F}(v)+(\bar{\mu} / \bar{\lambda}) \mathscr{G}(v))}{r_{n}} \\
& \leq \frac{\sup _{|t| \leq \xi_{n}}\left(T F(t)+(\bar{\mu} / \bar{\lambda}) \int_{0}^{T} G(x, t) \mathrm{d} x\right)}{r_{n}} \\
& \leq 2\left(\frac{C_{0}}{C_{1}}\right)^{2} \\
& \cdot\left[\frac{T \sup _{|t| \leq \xi_{n}} F(t)}{\xi_{n}^{2}}+\frac{\bar{\mu}}{\bar{\lambda}} \frac{\int_{0}^{T} \sup _{|t| \leq \xi_{n}} G(x, t) \mathrm{d} x}{\xi_{n}^{2}}\right] .
\end{aligned}
$$

Moreover, from assumptions (F3) and (G3), we have

$$
\begin{aligned}
& \lim _{n \rightarrow+\infty} \frac{T \sup _{|t| \leq \xi_{n}} F(t)}{\xi_{n}^{2}}+\frac{\bar{\mu}}{\bar{\lambda}} \lim _{n \rightarrow+\infty} \frac{\int_{0}^{T} \sup _{|t| \leq \xi_{n}} G(x, t) \mathrm{d} x}{\xi_{n}^{2}} \\
& \quad<+\infty
\end{aligned}
$$

which follows

$$
\lim _{n \rightarrow+\infty} \frac{\int_{0}^{T} \sup _{|t| \leq \xi_{n}} J(x, t) \mathrm{d} x}{\xi_{n}^{2}}<+\infty .
$$

Therefore,

$$
\begin{aligned}
\gamma & \leq \liminf _{n \rightarrow+\infty} \varphi\left(r_{n}\right) \\
& \leq 2\left(\frac{C_{0}}{C_{1}}\right)^{2} \liminf _{\xi \rightarrow+\infty} \frac{\int_{0}^{T} \sup _{|t| \leq \xi} J(x, t) \mathrm{d} x}{\xi^{2}}<+\infty .
\end{aligned}
$$

Since

$$
\begin{aligned}
\frac{\int_{0}^{T} \sup _{|t| \leq \xi} J(x, s) \mathrm{d} x}{\xi^{2}} \leq & \frac{T \sup _{|t| \leq \xi} F(t)}{\xi^{2}} \\
& +\frac{\bar{\mu}}{\bar{\lambda}} \frac{\int_{0}^{T} \sup _{|t| \leq \xi} G(x, t) \mathrm{d} x}{\xi^{2}}
\end{aligned}
$$

and taking (G3) into account, we get

$$
\begin{aligned}
& \liminf _{\xi \rightarrow+\infty} \frac{\int_{0}^{T} \sup _{|t| \leq \xi} J(x, t) \mathrm{d} x}{\xi^{2}} \\
& \quad \leq \liminf _{\xi \rightarrow+\infty} \frac{T \sup _{|t| \leq \xi} F(t)}{\xi^{2}}+\frac{\bar{\mu}}{\bar{\lambda}} G_{\infty} .
\end{aligned}
$$


Moreover, by assumption (G3) we have

$$
\begin{gathered}
\limsup _{\xi \rightarrow+\infty} \frac{\int_{0}^{T} J\left(x, \xi\left(x^{2}-T x\right)\right) d x}{D(\xi)} \\
\quad \geq \limsup _{\xi \rightarrow+\infty} \frac{\int_{0}^{T} F\left(\xi\left(x^{2}-T x\right)\right) d x}{D(\xi)} .
\end{gathered}
$$

Therefore, from (60) and (61), we observe that

$$
\begin{aligned}
\bar{\lambda} & \in\left(\nu_{1}, v_{2}\right) \\
& \subseteq\left(\frac{1}{\limsup _{\xi \rightarrow+\infty}\left(\int_{0}^{T} J\left(x, \xi\left(x^{2}-T x\right)\right) \mathrm{d} x / D(\xi)\right)},\right. \\
& \left.\frac{1}{2\left(C_{0} / C_{1}\right)^{2} \liminf _{\xi \rightarrow+\infty}\left(\int_{0}^{T} \sup _{|t| \leq \xi} J(x, t) \mathrm{d} x / \xi^{2}\right)}\right) \\
& \subseteq\left(0, \frac{1}{\gamma}\right) .
\end{aligned}
$$

For the fixed $\bar{\lambda}$, inequality (58) ensures that condition (b) of Theorem 7 can be applied and either $I_{-}$has a global minimum or there exists a sequence $\left\{u_{n}\right\}$ of weak solutions of problem (1) such that $\lim _{n \rightarrow \infty}\left\|u_{n}\right\|=+\infty$. Now we prove that for the fixed $\bar{\lambda}$ the functional $I_{\bar{\lambda}}$ has no global minimum. Let us verify that the functional $I_{\bar{\lambda}}$ is unbounded from below. Since

$$
\frac{1}{\bar{\lambda}}<\limsup _{\xi \rightarrow+\infty} \frac{\int_{0}^{T} F\left(\xi\left(x^{2}-T x\right)\right) \mathrm{d} x}{D(\xi)},
$$

there exist a sequence $\left\{\eta_{n}\right\}$ of positive numbers and a constant $\tau$ such that $\lim _{n \rightarrow+\infty} \eta_{n}=+\infty$ and

$$
\frac{1}{\bar{\lambda}}<\tau<\frac{\int_{0}^{T} F\left(\eta_{n}\left(x^{2}-T x\right)\right) \mathrm{d} x}{D\left(\eta_{n}\right)}
$$

for each $n \in \mathbb{N}$ large enough. For all $n \in \mathbb{N}$, put

$$
w_{n}(x):=\eta_{n}\left(x^{2}-T x\right) \text { for every } x \in[0, T] .
$$

For any fixed $n \in \mathbb{N}$, clearly $w_{n} \in X$ and one has

$$
\left\|w_{n}\right\|_{X}^{2} \leq C_{2}^{2}\left\|w_{n}\right\|^{2}=\eta_{n}^{2} C_{2}^{2}\left(4 T+\frac{T^{3}}{3}+\frac{T^{5}}{30}\right),
$$

and so

$$
\begin{aligned}
\mathcal{N}\left(w_{n}\right) \leq & \frac{C_{2}^{2}}{2} \eta_{n}^{2}\left(4 T+\frac{T^{3}}{3}+\frac{T^{5}}{30}\right) \\
& +\sum_{k=1}^{m} \int_{0}^{\eta_{n}\left(2 x_{k}-T\right)} I_{1 k}(s) \mathrm{d} s \\
& +\sum_{k=1}^{m} \int_{0}^{\eta_{n}\left(x_{k}^{2}-T x_{k}\right)} I_{2 k}(s) \mathrm{d} s=D\left(\eta_{n}\right) .
\end{aligned}
$$

By (64) and (67) and since $G$ is nonnegative we observe that

$$
\begin{aligned}
I_{\bar{\lambda}}\left(w_{n}\right) & =\mathcal{N}\left(w_{n}\right)-\bar{\lambda} \mathscr{M}\left(w_{n}\right) \\
& \leq D\left(\eta_{n}\right)-\bar{\lambda} \int_{0}^{T} F\left(\eta_{n}\left(x^{2}-T x\right)\right) \mathrm{d} x \\
& <D\left(\eta_{n}\right)(1-\bar{\lambda} \tau)
\end{aligned}
$$

for every $n \in \mathbb{N}$ large enough; since $\bar{\lambda} \tau>1$ and $\lim _{n \rightarrow+\infty} \eta_{n}=$ $+\infty$, it follows that

$$
\lim _{n \rightarrow+\infty} I_{\bar{\lambda}}\left(w_{n}\right)=-\infty
$$

So, the functional $I_{\bar{\lambda}}$ is unbounded from below, and it shows that $I \bar{\lambda}$ has no global minimum. Therefore, from part (b) of Theorem 7 , the functional $I_{\bar{\lambda}}$ admits a sequence of critical points $\left\{\bar{u}_{n}\right\} \subset X$ such that $\lim _{n \rightarrow+\infty} \mathcal{N}\left(\bar{u}_{n}\right)=+\infty$. Since $\mathcal{N}$ is bounded on bounded sets and taking into account that $\lim _{n \rightarrow+\infty} \mathcal{N}\left(\bar{u}_{n}\right)=+\infty$, then $\left\{\bar{u}_{n}\right\}$ has to be unbounded; that is,

$$
\lim _{n \rightarrow+\infty}\left\|\bar{u}_{n}\right\|_{X}=+\infty
$$

Also, if $\bar{u}_{n} \in X$ is a critical point of $I_{\bar{\lambda}}$, clearly, by definition, one has

$$
I_{\bar{\lambda}}^{\circ}\left(\bar{u}_{n}, v-\bar{u}_{n}\right) \geq 0, \quad \text { for every } v \in X .
$$

Finally, by Lemma 11, the critical points of $I_{\bar{\lambda}}$ are weak solutions for problem (1), and, by Lemma 10, every weak solution of (1) is a classical solution of (1). Hence, we have the result.

Remark 13. Under the conditions

$$
\begin{gathered}
\liminf _{\xi \rightarrow+\infty} \frac{\sup _{|t| \leq \xi} F(t)}{\xi^{2}}=0, \\
\limsup _{\xi \rightarrow+\infty} \frac{\int_{0}^{T} F\left(\xi\left(x^{2}-T x\right)\right) d x}{D(\xi)}=+\infty
\end{gathered}
$$

from Theorem 12, we see that, for every $\lambda>0$ and for each $\mu \epsilon$ $\left[0, C_{1}^{2} / 2 C_{0}^{2} G_{\infty}\right)$, problem (1) admits a sequence of solutions which is unbounded in $X$. Moreover, if $G_{\infty}=0$, the result holds for every $\lambda>0$ and $\mu \geq 0$.

The following result is a special case of Theorem 12 with $\mu=0$.

Theorem 14. Assume that (F1)-(F3) and (I1) hold. Then, for each

$$
\begin{aligned}
\lambda \in\left(\frac{1}{\limsup _{\xi \rightarrow+\infty}\left(\int_{0}^{T} F\left(\xi\left(x^{2}-T x\right)\right) \mathrm{d} x / D(\xi)\right)},\right. \\
\\
\left.\frac{1}{2 T\left(C_{0} / C_{1}\right)^{2} \lim \inf _{\xi \rightarrow+\infty}\left(\sup _{|t| \leq \xi} F(t) / \xi^{2}\right)}\right),
\end{aligned}
$$


the problem

$$
\begin{aligned}
& u^{(i v)}(x)-p u^{\prime \prime}(x)+a(x) u(x) \in \lambda \partial F(u(x)), \\
& x \in[0, T] \backslash Q, \\
& \Delta\left(u^{\prime \prime}\left(x_{k}\right)\right)=I_{1 k}\left(u^{\prime}\left(x_{k}\right)\right), \\
& -\Delta\left(u^{\prime \prime \prime}\left(x_{k}\right)\right)=I_{2 k}\left(u\left(x_{k}\right)\right), \\
& u(0)=u^{\prime \prime}(0)=u(T)=u^{\prime \prime}(T)=0, \ldots, m,
\end{aligned}
$$

has an unbounded sequence of classical solutions in $X$.

Now, we present the following example to illustrate Theorem 14.

Example 15. Let $T=1, p=1$, and $a(x)=1+\cos x$ for every $x \in \mathbb{R}$. Thus, $a$ is a continuous positive even $2 \pi$-periodic function on $\mathbb{R}$. Define the function

$$
F(s)= \begin{cases}0, & \text { if } s \in[-1,1] \\ s^{2}-1, & \text { if } s \in[-2,-1) \cup(1,2] \\ 3, & \text { if } s \in[-\infty,-2) \cup(2,+\infty) .\end{cases}
$$

Clearly, $F$ is a continuous convex function with $F(0)=0$. An easy calculation shows that

$$
\partial F(s)= \begin{cases}\{0\}, & \text { if } s \in(-\infty,-2), \\ {[-4,0],} & \text { if } s=-2, \\ (-4,-2), & \text { if } s \in(-2,-1), \\ {[-2,0],} & \text { if } s=-1, \\ {[0\},} & \text { if } s \in(-1,1), \\ {[0,2],} & \text { if } s=1, \\ {[2,4),} & \text { if } s \in(1,2) \\ \{0,4], & \text { if } s=2, \\ \{0\}, & \text { if } s \in(2,+\infty) .\end{cases}
$$

Hence, assumptions $(F 1)$ and (F2) hold. Moreover, let $Q=$ $\{1 / 3,2 / 3\}, I_{11}(x)=I_{12}(x)=(1 / 8) x$, and $I_{21}(x)=I_{22}(x)=$ $(2 / 5) x$ for every $x \in \mathbb{R}$. Thus, (I1) is satisfied and

$$
\begin{aligned}
D(\xi)= & \frac{131 C_{2}^{2}}{30} \xi^{2}+2 \sum_{k=1}^{2} \int_{0}^{\xi\left(2 x_{k}-1\right)} I_{1 k}(s) \mathrm{d} s \\
& +2 \sum_{k=1}^{2} \int_{0}^{\xi\left(x_{k}^{2}-x_{k}\right)} I_{2 k}(s) \mathrm{d} s=\frac{131 C_{2}^{2}}{30} \xi^{2}+\frac{17}{324}
\end{aligned}
$$

So, we have

$$
\begin{aligned}
& \liminf _{\xi \rightarrow+\infty} \frac{\sup _{|t| \leq \xi} F(t)}{\xi^{2}}=0, \\
& \limsup _{\xi \rightarrow+\infty} \frac{\int_{0}^{1} F\left(\xi x^{2}-\xi x\right) \mathrm{d} x}{D(\xi)} \\
& \quad \geq \lim _{\xi \rightarrow \infty} \frac{\xi^{2}-1}{\left(131 C_{2}^{2} / 30\right) \xi^{2}+17 / 324}=\frac{30}{131 C_{2}^{2}} .
\end{aligned}
$$

Therefore, by applying Theorem 14, the problem

$$
\begin{aligned}
& u^{(i v)}(x)-u^{\prime \prime}(x)+a(x) u(x) \in \lambda \partial F(u(x)), \\
& \Delta\left(u^{\prime \prime}\left(\frac{1}{3}\right)\right)=\frac{1}{8}\left(u^{\prime}\left(\frac{1}{3}\right)\right), \\
& -\Delta\left(u^{\prime \prime \prime}\left(\frac{1}{3}\right)\right)=\frac{2}{5}\left(u\left(\frac{1}{3}, \frac{2}{3}\right\}\right), \\
& \Delta\left(u^{\prime \prime}\left(\frac{2}{3}\right)\right)=\frac{1}{8}\left(u^{\prime}\left(\frac{2}{3}\right)\right), \\
& -\Delta\left(u^{\prime \prime \prime}\left(\frac{2}{3}\right)\right)=\frac{2}{5}\left(u\left(\frac{2}{3}\right)\right), \\
& u(0)=u^{\prime \prime}(0)=u(1)=u^{\prime \prime}(1)=0
\end{aligned}
$$

for $\lambda \in\left(0,30 / 131 C_{2}^{2}\right)$ has an unbounded sequence of classical solutions in $H^{2}(0,1) \cap H_{0}^{1}(0,1)$.

Now we state the following consequence of Theorem 14, using the following assumptions:

$$
\begin{aligned}
& (F 4) \lim \inf _{\xi \rightarrow+\infty}\left(\sup _{|t| \leq \xi} F(t) / \xi^{2}\right)<C_{1}^{2} / 2 T C_{0}^{2} \\
& (F 5) \lim \sup _{\xi \rightarrow+\infty}\left(\int_{0}^{T} F\left(\xi\left(x^{2}-T x\right)\right) \mathrm{d} x / D(\xi)\right)>1 .
\end{aligned}
$$

Corollary 16. Assume that (F1), (F2), (F4), (F5), and (I1) hold. Then, the problem

$$
\begin{aligned}
& u^{(i v)}(x)-p u^{\prime \prime}(x)+a(x) u(x) \in \partial F(u(x)), \\
& \left.x\left(u^{\prime \prime}\left(x_{k}\right)\right)=I_{1 k}\left(u^{\prime}\left(x_{k}\right)\right), T\right] \backslash Q, \\
& -\Delta\left(u^{\prime \prime \prime}\left(x_{k}\right)\right)=I_{2 k}\left(u\left(x_{k}\right)\right), \\
& \quad k=1,2, \ldots, m, \\
& u(0)=u^{\prime \prime}(0)=u(T)=u^{\prime \prime}(T)=0
\end{aligned}
$$

has an unbounded sequence of classical solutions in $X$.

Remark 17. Theorem 1 in Introduction is an immediate consequence of Corollary 16.

Here, we give a consequence of the main result. 
Corollary 18. Let $F_{1}: \mathbb{R} \rightarrow \mathbb{R}$ be a locally Lipschitz function such that $F_{1}(0)=0,-\partial F_{1}(-s)=\partial F_{1}(s),|\xi| \leq a\left(1+|s|^{r_{1}-1}\right)$ for all $s \in \mathbb{R}, \xi \in F_{1}(s), r_{1} \in[1,2)(a>0)$. Furthermore, suppose that

(C1) $\liminf _{\xi \rightarrow+\infty}\left(\sup _{|t| \leq \xi} F_{1}(t) / \xi^{2}\right)<+\infty$,

$(C 2) \lim \sup _{\xi \rightarrow+\infty}\left(\int_{0}^{T} F_{1}\left(\xi\left(x^{2}-T x\right)\right) \mathrm{d} x / D(\xi)\right)=+\infty$.

Then, for every function $F_{2}: \mathbb{R} \rightarrow \mathbb{R}$ which is locally Lipschitz function such that $F_{2}(0)=0,-\partial F_{2}(-s)=\partial F_{2}(s)$, and $|\xi| \leq$ $a\left(1+|s|^{r_{1}-1}\right)$ for all $s \in \mathbb{R}, \xi \in F_{2}(s), r_{1} \in[1,2)(a>0)$ and satisfies the conditions

$$
\begin{array}{r}
\sup _{t \in \mathbb{R}} F_{2}(t) \leq 0, \\
\liminf _{\xi \rightarrow+\infty} \frac{\int_{0}^{T} F_{2}\left(\xi\left(x^{2}-T x\right)\right) d x}{D(\xi)}>-\infty,
\end{array}
$$

for each

$$
\begin{aligned}
& \lambda \in(0, \\
& \left.\frac{1}{2 T\left(C_{0} / C_{1}\right)^{2} \liminf \inf _{\xi \rightarrow+\infty}\left(\sup _{|t| \leq \xi} F_{1}(t) / \xi^{2}\right)}\right),
\end{aligned}
$$

the problem

$$
\begin{aligned}
& u^{(i v)}(x)-p u^{\prime \prime}(x)+a(x) u(x) \\
& \quad \in \lambda\left(\partial F_{1}(u(x))+\partial F_{2}(u(x))\right), \quad x \in[0, T] \backslash Q, \\
& \Delta\left(u^{\prime \prime}\left(x_{k}\right)\right)=I_{1 k}\left(u^{\prime}\left(x_{k}\right)\right), \\
& -\Delta\left(u^{\prime \prime \prime}\left(x_{k}\right)\right)=I_{2 k}\left(u\left(x_{k}\right)\right), \\
& u(0)=u^{\prime \prime}(0)=u(T)=u^{\prime \prime}(T)=0
\end{aligned}
$$

has an unbounded sequence of classical solutions in X.

Proof. Set $F(t)=F_{1}(t)+F_{2}(t)$ for all $t \in \mathbb{R}$. Assumption (C2) along with the condition

$$
\liminf _{\xi \rightarrow+\infty} \frac{\int_{0}^{T} F_{2}\left(\xi\left(x^{2}-T x\right)\right) d x}{D(\xi)}>-\infty
$$

yields

$$
\begin{aligned}
& \limsup _{\xi \rightarrow+\infty} \frac{\int_{0}^{T} F\left(\xi\left(x^{2}-T x\right)\right) \mathrm{d} x}{D(\xi)} \\
& =\limsup _{\xi \rightarrow+\infty} \frac{\int_{0}^{T} F_{1}\left(\xi\left(x^{2}-T x\right)\right) \mathrm{d} x+\int_{0}^{T} F_{2}\left(\xi\left(x^{2}-T x\right)\right) \mathrm{d} x}{D(\xi)} \\
& =+\infty .
\end{aligned}
$$

Moreover, assumption (C1) together with the condition

$$
\sup _{t \in \mathbb{R}} F_{2}(t) \leq 0
$$

ensures

$$
\liminf _{\xi \rightarrow+\infty} \frac{\sup _{|t| \leq \xi} F(t)}{\xi^{2}} \leq \liminf _{\xi \rightarrow+\infty} \frac{\sup _{|t| \leq \xi} F_{1}(t)}{\xi^{2}}<+\infty .
$$

Since

$$
\begin{aligned}
& \frac{1}{\liminf _{\xi \rightarrow+\infty}\left(\sup _{|t| \leq \xi} F(t) / \xi^{2}\right)} \\
& \geq \frac{1}{\liminf _{\xi \rightarrow+\infty}\left(\sup _{|t| \leq \xi} F_{1}(t) / \xi^{2}\right)},
\end{aligned}
$$

by applying Theorem 14, we have the desired conclusion.

Remark 19. We observe that in Theorem 12 we can replace $\xi \rightarrow+\infty$ with $\xi \rightarrow 0^{+}$, and then by the same argument as in the proof of Theorem 12, but using conclusion (c) of Theorem 7 instead of (b), problem (1) has a sequence of pairwise distinct classical solutions, which strongly converges to 0 in $X$.

We end this paper by presenting the following example.

Example 20. Let $T=\pi, p=1$, and $a(x)=|\sin x|+1$ for every $x \in \mathbb{R}$. Thus, $a$ is a continuous positive even $2 \pi$ periodic function on $\mathbb{R}$. Now let $F(s)=-|s|$ for all $s \in \mathbb{R}$ and $G(x, s)=x(1-\cos s)$ for all $(x, s) \in[0, \pi] \times \mathbb{R}$. Hence, $F$ is locally Lipschitz function and

$$
\partial F(s)= \begin{cases}\{1\}, & \text { if } s<0 \\ {[-1,1],} & \text { if } s=0 \\ \{-1\}, & \text { if } s>0\end{cases}
$$

Therefore, $(F 1)$ and $(F 2)$ hold, and also we can simply see that $(G 1)$ and (G2) hold. Moreover, let $Q=\{\pi / 3,2 \pi / 3\}, I_{11}(x)=$ $I_{12}(x)=x(1+x)$, and $I_{21}(x)=I_{22}(x)=x^{5}$ for every $x \in \mathbb{R}$. Thus, (I1) is satisfied. An easy computation shows that

$$
\begin{aligned}
\frac{\int_{0}^{\pi} F\left(\xi\left(x^{2}-x\right)\right) \mathrm{d} x}{D(\xi)} & =\frac{\xi\left(\int_{0}^{1}\left(x-x^{2}\right) \mathrm{d} x+\int_{1}^{\pi}\left(x^{2}-x\right) \mathrm{d} x\right)}{\left(C_{2}^{2} / 2\right) \xi^{2}\left(4 \pi+\pi^{3} / 3+\pi^{5} / 30\right)+2 \sum_{k=1}^{2}\left(\int_{0}^{\xi\left(2 x_{k}-\pi\right)} I_{1 k}(s)+\int_{0}^{\xi\left(x_{k}^{2}-\pi x_{k}\right)} I_{2 k}(s) \mathrm{d} s\right)} \\
& =\frac{\xi\left(\pi^{3} / 3-\pi^{2} / 2+1 / 3\right)}{\left(C_{2}^{2} / 2\right) \xi^{2}\left(4 \pi+\pi^{3} / 3+\pi^{5} / 30\right)+\left(2^{7} / 9^{6}\right) \pi^{12} \xi^{6}}
\end{aligned}
$$


for some $\xi \in \mathbb{R}$. So,

$$
\begin{gathered}
\liminf _{\xi \rightarrow 0^{+}} \frac{\sup _{|t| \leq \xi} F(t)}{\xi^{2}}=0, \\
\limsup _{\xi \rightarrow 0^{+}} \frac{\int_{0}^{\pi} F\left(\xi x^{2}-\xi x\right) \mathrm{d} x}{D(\xi)}=+\infty .
\end{gathered}
$$

Also

$$
\begin{aligned}
& \int_{0}^{\pi} x(1-\cos t) \mathrm{d} x=\frac{\pi^{2}}{2}(1-\cos t) \geq 0 \quad \forall t \in \mathbb{R}, \\
& \lim _{\xi \rightarrow+0^{+}} \frac{\sup _{|t| \leq \xi} \int_{0}^{\pi} x(1-\cos t) \mathrm{d} x}{\xi^{2}} \\
& \quad=\frac{\pi^{2}}{2} \lim _{\xi \rightarrow+0^{+}} \frac{\sup _{|t| \leq \xi}(1-\cos t)}{\xi^{2}}=\frac{\pi^{2}}{4} .
\end{aligned}
$$

Hence, taking Remark 19 into account, the problem

$$
\begin{aligned}
& u^{(i v)}(x)-u^{\prime \prime}(x)+(2+\sin x) u(x) \\
& \in \lambda \partial F(u(x))+\mu \partial G_{u}(x, u), \\
& \quad x \in[0, \pi] \backslash\left\{\frac{\pi}{3}, \frac{2 \pi}{3}\right\}, \\
& \Delta\left(u^{\prime \prime}\left(\frac{\pi}{3}\right)\right)=u^{\prime}\left(\frac{\pi}{3}\right)\left(1+u^{\prime}\left(\frac{\pi}{3}\right)\right), \\
& -\Delta\left(u^{\prime \prime \prime}\left(\frac{\pi}{3}\right)\right)=u^{5}\left(\frac{\pi}{3}\right), \\
& \Delta\left(u^{\prime \prime}\left(\frac{2 \pi}{3}\right)\right)=u^{\prime}\left(\frac{2 \pi}{3}\right)\left(1+u^{\prime}\left(\frac{2 \pi}{3}\right)\right), \\
& -\Delta\left(u^{\prime \prime \prime}\left(\frac{2 \pi}{3}\right)\right)=u^{5}\left(\frac{2 \pi}{3}\right), \\
& u(0)=u^{\prime \prime}(0)=u(\pi)=u^{\prime \prime}(\pi)=0
\end{aligned}
$$

for $\lambda \in(0, \infty)$ and $\mu \in\left[0,2 C_{1}^{2} / C_{0}^{2} \pi^{3}\right)$ has an unbounded sequence of pairwise distinct classical solutions in $H^{2}(0, \pi) \cap$ $H_{0}^{1}(0, \pi)$.

\section{Conflict of Interests}

The authors declare that there is no conflict of interests regarding the publication of this paper.

\section{References}

[1] G. A. Afrouzi, S. Heidarkhani, and D. O'Regan, "Existence of three solutions for a doubly eigen-value fourth-order boundary value problem," Taiwanese Journal of Mathematics, vol. 15, no. 1, pp. 201-210, 2011.

[2] G. Bonanno and B. Di Bella, "A boundary value problem for fourth-order elastic beam equations," Journal of Mathematical Analysis and Applications, vol. 343, no. 2, pp. 1166-1176, 2008.

[3] J. R. Graef and B. Yang, "Existnence and nonexistence of positive solutions of fourth order nonlinear boundary value problems," Applicable Analysis, vol. 74, no. 1-2, pp. 201-214, 2000.
[4] R. Ma, "Multiple positive solutions for a semipositone fourthorder boundary value problem," Hiroshima Mathematical Journal, vol. 33, no. 2, pp. 217-227, 2003.

[5] D. Bainov and P. Simeonov, Systems with Impulse Effect, Ellis Horwood Series: Mathematics and Its Applications, Ellis Horwood, Chichester, UK, 1989.

[6] M. Benchohra, J. Henderson, and S. Ntouyas, Theory of Impulsive Differential Equations, vol. 2 of Contemporary Mathematics and Its Applications, Hindawi Publishing Corporation, New York, NY, USA, 2006.

[7] T. E. Carter, "Necessary and sufficient conditions for optimal impulsive rendezvous with linear equations of motion," Dynamics and Control, vol. 10, no. 3, pp. 219-227, 2000.

[8] S. Heidarkhani, M. Ferrara, and A. Salari, "Infinitely many periodic solutions for a class of perturbed second-order differential equations with impulses," Acta Applicandae Mathematicae, vol. 139, pp. 81-94, 2015.

[9] X. Liu and A. R. Willms, "Impulsive controllability of linear dynamical systems with applications to maneuvers of spacecraft," Mathematical Problems in Engineering, vol. 2, no. 4, pp. 277-299, 1996.

[10] A. Cabada and S. Tersian, "Existence and multiplicity of solutions to boundary value problems for fourth-order impulsive differential equations," Boundary Value Problems, vol. 2014, article 105, 2014.

[11] J. Sun, H. Chen, and L. Yang, "Variational methods to fourthorder impulsive differential equations," Journal of Applied Mathematics and Computing, vol. 35, no. 1-2, pp. 323-340, 2011.

[12] J. Xie and Z. Luo, "Solutions to a boundary value problem of a fourth-order impulsive differential equation," Boundary Value Problems, vol. 2013, article 154, 14 pages, 2013.

[13] S. Heidarkhani, G. A. Afrouzi, A. Hadjian, and J. Henderson, "Existence of infinitely many anti-periodic solutions for secondorder impulsive differential inclusions," Electronic Journal of Differential Equations, vol. 2013, article 97, 13 pages, 2013.

[14] A. Iannizzotto, “Three critical points for perturbed nonsmooth functionals and applications," Nonlinear Analysis: Theory, Methods \& Applications, vol. 72, no. 3-4, pp. 1319-1338, 2010.

[15] A. Iannizzotto, "Three periodic solutions for an ordinary differential inclusion with two parameters," Annales Polonici Mathematici, vol. 103, no. 1, pp. 89-100, 2012.

[16] A. Kristály, "Infinitely many solutions for a differential inclusion problem in $\mathbb{R}^{N}$," Journal of Differential Equations, vol. 220, no. 2, pp. 511-530, 2006.

[17] A. Kristály, W. Marzantowicz, and C. Varga, "A non-smooth three critical points theorem with applications in differential inclusions," Journal of Global Optimization, vol. 46, no. 1, pp. 49-62, 2010.

[18] Y. Tian and J. Henderson, "Three anti-periodic solutions for second-order impulsive differential inclusions via nonsmooth critical point theory," Nonlinear Analysis, vol. 75, no. 18, pp. 6496-6505, 2012.

[19] B.-X. Yang and H.-R. Sun, "Periodic solutions for semilinear fourth-order differential inclusions via nonsmooth critical point theory," Journal of Function Spaces, vol. 2014, Article ID 816490, 6 pages, 2014.

[20] B. Ricceri, "A general variational principle and some of its applications," Journal of Computational and Applied Mathematics, vol. 113, no. 1-2, pp. 401-410, 2000. 
[21] S. A. Marano and D. Motreanu, "Infinitely many critical points of non-differentiable functions and applications to a Neumanntype problem involving the $p$-Laplacian," Journal of Differential Equations, vol. 182, no. 1, pp. 108-120, 2002.

[22] G. Bonanno and G. M. Bisci, "Infinitely many solutions for a boundary value problem with discontinuous nonlinearities," Boundary Value Problems, vol. 2009, Article ID 670675, 2009.

[23] L. H. Erbe and W. Krawcewicz, "Existence of solutions to boundary value problems for impulsive second order differential inclusions," The Rocky Mountain Journal of Mathematics, vol. 22, no. 2, pp. 519-539, 1992.

[24] D. Motreanu and P. D. Panagiotopoulos, Minimax Theorems and Qualitative Properties of the Solutions of Hemivariational Inequalities, vol. 29 of Nonconvex Optimization and Its Applications, Kluwer Academic Publishers, Dordrecht, The Netherlands, 1999.

[25] F. H. Clarke, Optimization and Nonsmooth Analysis, Canadian Mathematical Society Series of Monographs and Advanced Texts, John Wiley \& Sons, New York, NY, USA, 1983.

[26] S. Tersian and J. Chaparova, "Periodic and homoclinic solutions of extended Fisher-Kolmogorov equations," Journal of Mathematical Analysis and Applications, vol. 260, no. 2, pp. 490-506, 2001.

[27] K. C. Chang, "Variational methods for nondifferentiable functionals and their applications to partial differential equations," Journal of Mathematical Analysis and Applications, vol. 80, no. 1, pp. 102-129, 1981. 


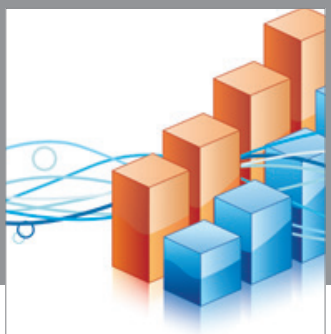

Advances in

Operations Research

vatem alat4

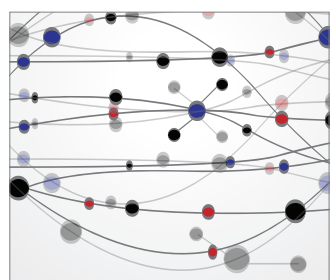

\section{The Scientific} World Journal
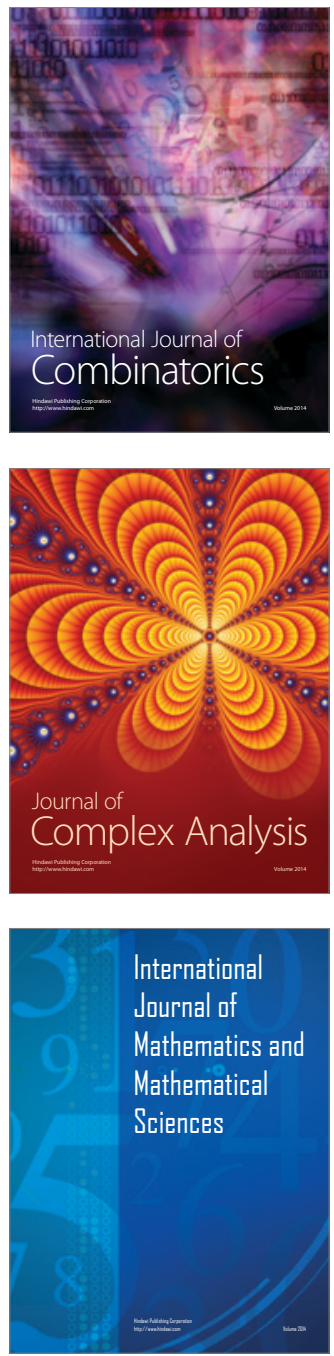
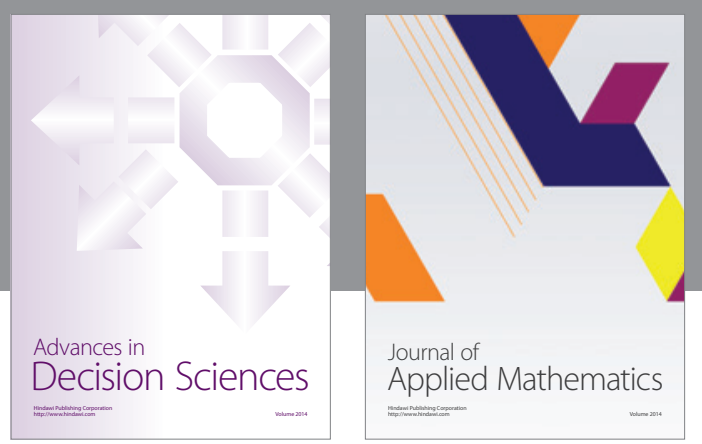

Algebra

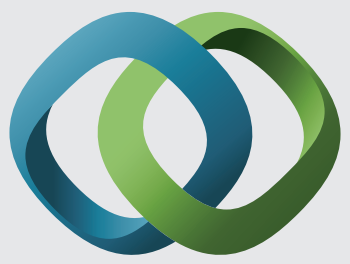

\section{Hindawi}

Submit your manuscripts at

http://www.hindawi.com
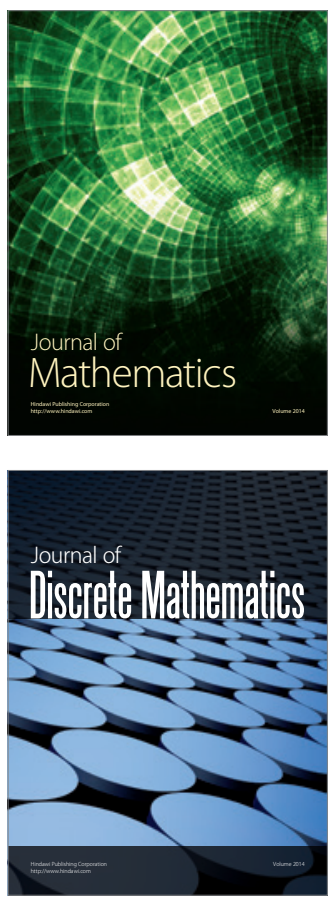

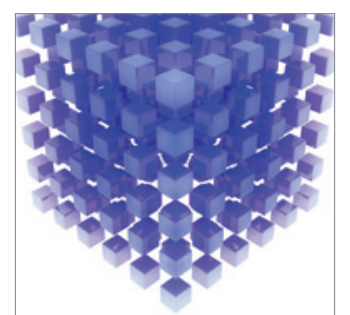

Mathematical Problems in Engineering
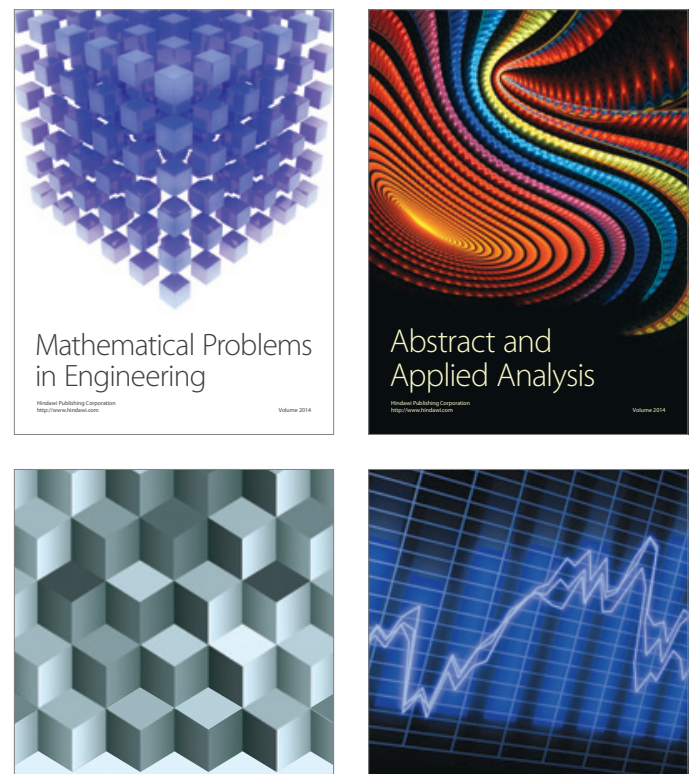

Journal of

Function Spaces

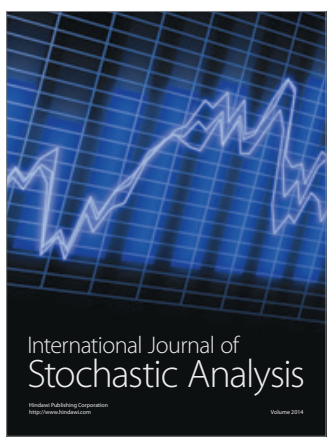

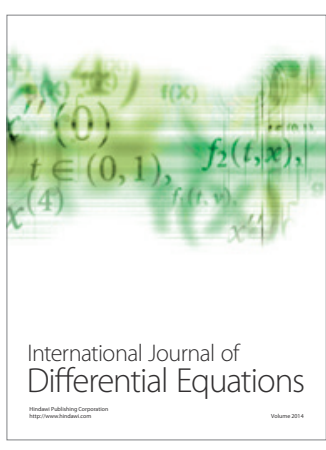
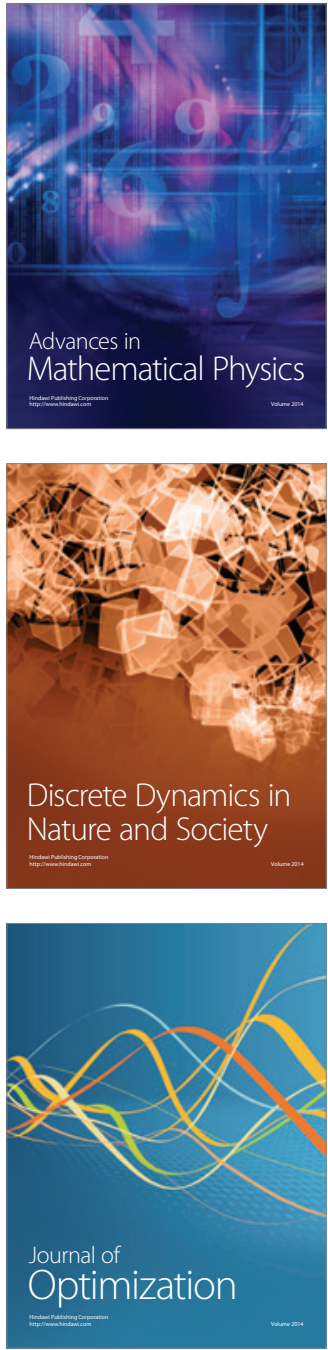